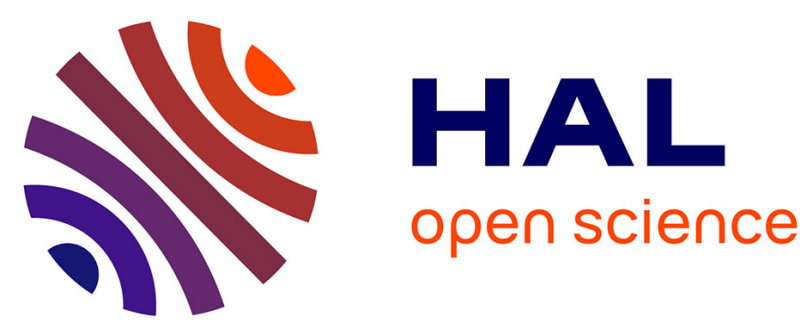

\title{
Résistance des sols aux maladies : XIII - Rôle des Fusarium oxysporum non pathogènes dans les mécanismes de résistance d'un sol de Noirmoutier aux fusarioses vasculaires \\ Giacomo Tamietti, Claude Alabouvette
}

\section{To cite this version:}

Giacomo Tamietti, Claude Alabouvette. Résistance des sols aux maladies: XIII - Rôle des Fusarium oxysporum non pathogènes dans les mécanismes de résistance d'un sol de Noirmoutier aux fusarioses vasculaires. Agronomie, 1986, 6 (6), pp.541-548. hal-00884908

\section{HAL Id: hal-00884908 \\ https://hal.science/hal-00884908}

Submitted on 1 Jan 1986

HAL is a multi-disciplinary open access archive for the deposit and dissemination of scientific research documents, whether they are published or not. The documents may come from teaching and research institutions in France or abroad, or from public or private research centers.
L'archive ouverte pluridisciplinaire HAL, est destinée au dépôt et à la diffusion de documents scientifiques de niveau recherche, publiés ou non, émanant des établissements d'enseignement et de recherche français ou étrangers, des laboratoires publics ou privés. 


\title{
Résistance des sols aux maladies : XIII - Rôle des Fusarium oxysporum non pathogènes dans les mécanismes de résistance d'un sol de Noir- moutier aux fusarioses vasculaires
}

\author{
Giacomo TAMIETTI \& Claude ALABOUVETTE $(*)$ \\ Instituto di Patologia Vegetale. Università di Torino, Via Pietro Giuria 15, Italie \\ (*) I.N.R.A., Station de Recherches sur la Flore pathogène dans le Sol, 17, rue Sully, F 21034 Dijon Cedex
}

L'emploi de la méthode standardisée d'évaluation du niveau de réceptivité des sols aux fusarioses vasculaires a permis de mettre en évidence le très fort niveau de résistance d'un sol maraîcher de Noirmoutier.

Cette résistance, détruite par traitement thermique du sol $\left(60^{\circ} \mathrm{C}, 30 \mathrm{mn}\right)$ et transmissible par simple mélange à un autre sol, est fondamentalement de nature microbiologique.

L'introduction de fortes populations de Pseudomonas fluorescents ne permet pas de rétablir la résistance dans le sol traité à la chaleur et l'apport de Fe E.D.T.A. est sans effet sur le niveau de résistance de ce sol. Il est done permis de conclure que les Pseudomonas fluorescents entrant en compétition pour le fer ne jouent qu'un rôle négligeable dans les mécanismes de résistance de ce sol. Au contraire, l'apport de Fusarium oxysporum non pathogènes suffit à induire un niveau élevé de résistance dans le sol traité à la chaleur. Comme l'addition de glucose induit une faible baisse du niveau de résistance du sol, il est vraisemblable que les mécanismes de résistance sont en relation avec des phénomènes de compétition nutritive comme dans le cas du sol résistant de Châteaurenard.

Trente souches de chacune des espèces $F$. oxysporum, $F$. roseum et $F$. solani ont été comparées pour leur aptitude à induire la résistance dans le sol traité à la chaleur. Globalement les $F$. oxysporum sont nettement plus efficaces, mais il n'existe pas de relation claire entre l'aptitude des souches à coloniser le sol ou la racine des plantes et leur aptitude à rendre le sol résistant. De plus, certaines souches ont permis d'induire des réactions de prémunition chez la tomate. Il est donc vraisemblable que les $F$. oxysporum non pathogènes disposent de différents mécanismes d'induction de résistance.

Mots clés additionnels : Pseudomonas fluorescents, compétition nutritive, fer, glucose, colonisation du sol, colonisation racinaire, prémunition. in the wilt-suppression mechanisms of a soil from Noirmoutier.

The high level of suppressiveness of a soil from Noirmoutier (Western France) has been established, using the standardized method to evaluate the level of soil receptivity to fusarium wilts. This suppressiveness was destroyed by heat treatment of the soil and was restored by mixing $10 \%$ of suppressive soil into the steamed suppressive soil, indicating that the suppressiveness is based on microbiological interactions. Introduction of high populations of fluorescent Pseudomonas isolated from this soil did not establish suppressiveness in steamed soil. Addition of Fe E.D.T.A. at $200 \mu \mathrm{g} / \mathrm{g}$ soil did not reduce the high level of suppressiveness of the suppressive soil. Thus, the role of fluorescent Pseudomonas competing for iron seems to be of low importance in this suppressive soil. On the other hand, addition of non-pathogenic strains of Fusarium oxysporum induced a high level of suppressiveness in steamed soil. As the addition of glucose slightly reduced the level of suppressiveness it could be assumed that the mechanism of suppression is similar to that previously described in the suppressive soil from Châteaurenard.

Thirty strains of each species $F$. oxysporum, $F$. roseum and $F$. solani were compared for their ability to induce suppressiveness in steamed soil. Overall, $F$. oxysporum were more effective than $F$. roseum and $F$. solani. Thus, non-pathogenic $F$. oxysporum seemed to be involved in this mechanism of suppression but their mode of action is not known. There was no clear relationship between the level of soil colonization or root colonization by different strains of $F$. oxysporum and their ability to induce suppressiveness. Furthermore, some strains were able to produce cross-protection in tomatoes indicating that these non-pathogenic $F$. oxysporum could be active through different modes of action.

Additional key words : Fluorescent Pseudomonas, competition for nutrients, iron, glucose, soil colonization, root colonization, cross protection. 


\section{INTRODUCTION}

La résistance des sols aux maladies d'origine tellurique est un phénomène observé depuis longtemps ; mais c'est seulement au cours de la dernière décennie que des recherches spécifiques y ont été consacrées (SCHNEIDER, 1982 ; COOK \& BAKER, 1983).

Les premiers travaux ayant pour objet la résistance des sols aux fusarioses vasculaires (STOVER, 1956; STOTZKY, 1967) ont attiré l'attention sur le rôle de certains facteurs abiotiques et, en particulier, des argiles de type montmorillonite. D'autres, plus récents, ont apporté la preuve que ces résistances reposent fondamentalement sur des interactions microbiennes qui inhibent l'activité de l'agent pathogène (LOUVET et al., 1976 ; SCHER \& BAKER, 1980). De très nombreux microorganismes antagonistes ont été décrits (ARJUNARAO, 1971 ; TU \& CHENG, 1981) mais les résultats les plus probants montrent l'importance du rôle joué soit par les Fusarium non pathogènes, soit par les Pseudomonas fluorescents.

Il a été démontré, en effet, que la présence de Fusarium oxysporum et $F$. solani non pathogènes est indispensable à l'expression de la résistance des sols de Châteaurenard qui repose sur des mécanismes de compétition nutritive (ROUXEL et al., 1979 ; ALABOUVETTE et al., 1985a). D'autre part, la résistance des sols de Salinas Valley met en jeu des relations de compétition pour le fer entre le $F$. oxysporum pathogène et la population de Pseudomonas fluorescents abondants dans la rhizosphère (SCHER \& BAKER, 1982).
D'un point de vue théorique, ces mécanismes mis en évidence dans des sols différents n'ont pas à être opposés ; ils peuvent au contraire se manifester conjointement dans un même sol. L'étude d'autres sols résistants aux fusarioses vasculaires est le moyen d'acquérir de nouveaux résultats permettant de comparer la validité des hypothèses émises précédemment.

La mise en évidence d'un nouveau sol résistant aux fusarioses vasculaires, dans l'île de Noirmoutier (France), a permis de réaliser une étude comparative des différents mécanismes microbiologiques susceptibles d'expliquer cette résistance. Cet article a pour but de rendre compte des résultats obtenus.

\section{MATÉRIEL ET MÉTHODES}

\section{A. Les sols}

L'étude porte sur un sol de Noirmoutier d'origine marine. L'échantillon étudié est prélevé dans une parcelle consacrée à la culture de pommes de terre de primeur. Ses caractéristiques agronomiques, ainsi que celles d'un échantillon du sol résistant de Châteaurenard utilisé comme témoin, sont présentées dans le tableau 1.

TABLEAU 1

Caractéristiques physico-chimiques et agronomiques des sols de Châteaurenard et Noirmoutier utilisés au cours de cette étude. Physico-chemical and agronomical properties of soils from Chateaurenard and Noirmoutier used in this study.

\begin{tabular}{|c|c|c|c|c|}
\hline Granulométrie & & & Châteaurenard & Noirmoutier \\
\hline Sables grossiers $\left(\begin{array}{lll}2 & -0,2 & \mathrm{~mm}\end{array}\right)$ & & p. 1000 & 5 & 531 \\
\hline Sables fins $\quad(0,2-0,05 \mathrm{~mm})$ & & p. 1000 & 98 & 119 \\
\hline Limon grossier $(0,05-0,02 \mathrm{~mm})$ & & p. 1000 & 192 & 33 \\
\hline Limon $\quad(0,02-0,002 \mathrm{~mm})$ & & p. 1000 & 152 & 49 \\
\hline Argile $\quad(<0,002 \mathrm{~mm})$ & & p. 1000 & 227 & 104 \\
\hline $\mathrm{pH}$ cau & & & 7,9 & 7,9 \\
\hline C organique (méthode Anne) & & p. 1000 & 22,6 & 13,7 \\
\hline Matières organiques & & p. 1000 & 38,8 & 23,5 \\
\hline N Kjeldahl & & p. 1000 & 2,93 & 1,79 \\
\hline $\mathrm{C} / \mathrm{N}$ & & & 7,71 & 7,65 \\
\hline Calcaire total & & p. 1000 & 318 & 162 \\
\hline Calcaires actifs & & p. 1000 & 92 & 7 \\
\hline Acide phosphorique & & p. 1000 & 0,29 & 0,48 \\
\hline Capacité d'échange & $\mathbf{M}$ eq. & p. 100 & 12,7 & 6,6 \\
\hline Ca échangeable & $\mathrm{M}$ eq. & p. $\quad 100$ & 37,4 & 28,1 \\
\hline $\mathrm{Mg}$ échangeable & M eq. & p. 100 & 1,96 & 1,12 \\
\hline $\mathrm{K}$ échangeable & M eq. & p. 100 & 0,915 & 0,855 \\
\hline Mn échangeable & & p.p.m. & 3,3 & 1,0 \\
\hline Al échangeable & $\mathbf{M}$ eq. & p. 100 & 0,2 & 0,1 \\
\hline Fe soluble & & p. $\quad 100$ & 0,76 & 0,28 \\
\hline Ca total & & p. 100 & 13,13 & 5,85 \\
\hline Mg total & & p. 100 & 0,80 & 0,75 \\
\hline $\mathrm{K}$ total & & p. 100 & 0,96 & 1,17 \\
\hline $\mathrm{Na}$ total & & p. 100 & 0,65 & 0,36 \\
\hline $\mathrm{Cu}$ total & & p.p.m. & 43,5 & 3,0 \\
\hline Fe total & & p. 100 & 2,19 & 1,0 \\
\hline Mn total & & p.p.m. & 623 & 178 \\
\hline Al total & & p. 100 & 4,59 & 2,53 \\
\hline $\mathrm{Cu}$ extractible E.D.T.A. & & p.p.m. & 14,2 & 0,9 \\
\hline Mn extractible E.D.T.A. & & p.p.m. & 13,3 & 5,1 \\
\hline Zn extractible E.D.T.A. & & p.p.m. & 2,4 & 4,6 \\
\hline
\end{tabular}


Dans certaines expériences le sol de Noirmoutier est utilisé après une désinfection plus ou moins poussée, réalisée par traitement thermique à l'autoclave à 60 , 80 ou $100{ }^{\circ} \mathrm{C}$ pendant $30 \mathrm{mn}$. Dans d'autres expériences, le sol est utilisé après amendement avec le glucose mélangé à sec à raison de $10 \mathrm{mg} / \mathrm{g}$ de sol ou avec le Fe-E.D.T.A. apporté en solution selon le procédé décrit par SCHER \& BAKER (1982). Cent $\mu \mathrm{g} / \mathrm{g}$ de solution sont pulvérisés à la surface du sol au moment du semis des plantes puis, une seconde fois, une semaine plus tard.

\section{B. Analyses microbiologiques}

Les analyses microbiologiques de la microflore du sol sont réalisées par étalement d'une suspensiondilution de sol sur milieu malt gélosé acidifié par l'acide citrique pour les champignons et sur le milieu B de King pour les bactéries et actinomycètes. L'analyse qualitative des populations de Fusarium est effectuée sur le milieu de KOMADA (1975) selon la technique d'incorporation directe de l'échantillon de sol décrite par ROUXEL \& BOUHOT (1971).

L'analyse de la mycoflore colonisant la racine est réalisée à partir de plantules de lin âgées de $18 \mathrm{j}$, cultivées en conditions contrôlées dans les sols étudiés. Les racines, lavées, sont désinfectées superficiellement par trempage pendant $5 \mathrm{mn}$ dans une solution d'hypochlorite de $\mathrm{Na}$ à 0,21 p. 100 de chlore actif puis déposées, entières, sur un milieu malt acidifié par l'acide citrique. Le dénombrement des colonies fongiques, puis leur détermination sont effectués respectivement après 36 et $72 \mathrm{~h}$ d'incubation.

\section{Etude de l'aptitude des souches de microorga- nismes à rendre le sol résistant}

Sept souches de Pseudomonas fluorescents isolées du sol de Noirmoutier, purifiées par clonage, sont cultivées sur milieu $\mathrm{B}$ de King pendant $24 \mathrm{~h}$ à $27^{\circ} \mathrm{C}$. Une suspension de bactéries est préparée par lavage de chaque culture avec $10 \mathrm{ml}$ d'eau stérile. Les suspensions obtenues sont mélangées à volume égal puis introduites à raison de $5 \mathrm{ml}$ par godet de $500 \mathrm{ml}$ dans le sol de Noirmoutier préalablement traité à la chaleur $\left(100^{\circ} \mathrm{C}\right.$, $30 \mathrm{mn}$ ) et immédiatement infesté artificiellement avec $F$. oxysporum f. sp. lini. Après $24 \mathrm{~h}$ d'incubation, la densité de Pseudomonas est déterminée par analyse microbiologique et le semis de lin est effectué avec des graines de la variété « Hera ». Le niveau de réceptivité des sols aux fusarioses vasculaires est déterminé suivant la technique précédemment décrite (ALABOUVETTE et al., 1982).

Quatre-vingt-dix souches - 30 de chacune des espèces $F$. oxysporum, $F$. roseum et $F$. solani isolées du sol résistant de Noirmoutier sur milieu de KOMADA sont cultivées sur milieu malt. Une suspension de conidies est réalisée à partir de chaque culture puis introduite à raison de $5 \mathrm{ml} / \mathrm{l}$ de sol préalablement traité à la chaleur $\left(100^{\circ} \mathrm{C}, 30 \mathrm{mn}\right)$. Après 3 semaines d'incubation à $25^{\circ} \mathrm{C}$, lorsque les Fusarium ont atteint leur développement maximum, la densité de population est déterminée par analyse microbiologique.
F. oxysporum f. sp. lini est alors introduit à la dose de 2000 germes/g de sol. Le semis est effectué avec le lin var. «Hera » et les plantes sont cultivées en serre à une température minimale de $18^{\circ} \mathrm{C}$. La mortalité des plantes est notée au cours des 6 semaines suivantes, chaque traitement comprenant 2 godets de 15 plantes chacun.

Dans une autre expérimentation, 5 souches de $F$. oxysporum non pathogènes sont cultivées, puis incubées dans le sol comme indiqué ci-dessus. Les 5 échantillons de sol sont alors mélangés, la densité des populations de Fusarium est déterminée, puis l'agent pathogène, $F$. oxysporum f. sp. lini, est introduit à différentes concentrations afin de déterminer le niveau de réceptivité des sols selon la technique précédemment décrite.

\section{Etude de l'aptitude de différentes souches de Fusarium à induire des réactions de résistance des plantes}

Pour l'étude de l'aptitude des souches de $F$. oxysporum à induire des réactions de résistance des plantes (phénomène de prémunition ou protection croisée), 9 souches de $F$. oxysporum non pathogènes et 4 souches de $F$. oxysporum pathogènes appartenant aux formes spéciales dianthi, lini, melonis, vasinfectum sont cultivées pendant une semaine en milieu malt liquide agité à $25^{\circ} \mathrm{C}$. Les propagules sont recueillies par centrifugation, lavées, puis remises en suspension de manière à obtenir une concentration de $1.10^{8}$ propagules $/ \mathrm{ml}$. Les racines de 30 plantules de tomate, var. "Marmande », âgées de $12 \mathrm{j}$, cultivées sur perlite, sont alors immergées pendant $15 \mathrm{~h}$ à $20^{\circ} \mathrm{C}$ dans les suspensions de conidies des souches étudiées puis 20 d'entre elles sont transférées pendant $1 \mathrm{~h}$ dans une suspension de conidies de la souche pathogène, $F$. oxysporum f. sp. lycopersici, les 10 autres constituant les témoins sont immergées dans l'eau. Les plantules sont alors repiquées dans un sol préalablement traité à la chaleur $\left(100^{\circ} \mathrm{C}, 30 \mathrm{mn}\right)$ et placées en conditions contrôlées $\left(20-25^{\circ} \mathrm{C}\right)$ en chambre de culture. Le nombre de plantes ayant survécu au repiquage est noté après 1 semaine de culture et l'apparition des symptômes de fusariose est suivie pendant 10 semaines. La notation finale est réalisée en sectionnant la base de la tige de façon à observer les symptômes internes de brunissement vasculaire.

\section{RÉSULTATS}

\section{A. Mise en évidence de la résistance du sol de Noirmoutier}

La résistance de ce sol aux fusarioses vasculaires, observée dans les cultures de la région de Noirmoutier, a été confirmée au laboratoire.

Les résultats présentés dans la figure 1 indiquent clairement que la résistance de ce sol de Noirmoutier est très élevée, supérieure à celle du sol de Châteaurenard utilisé comme témoin dans cet essai. 


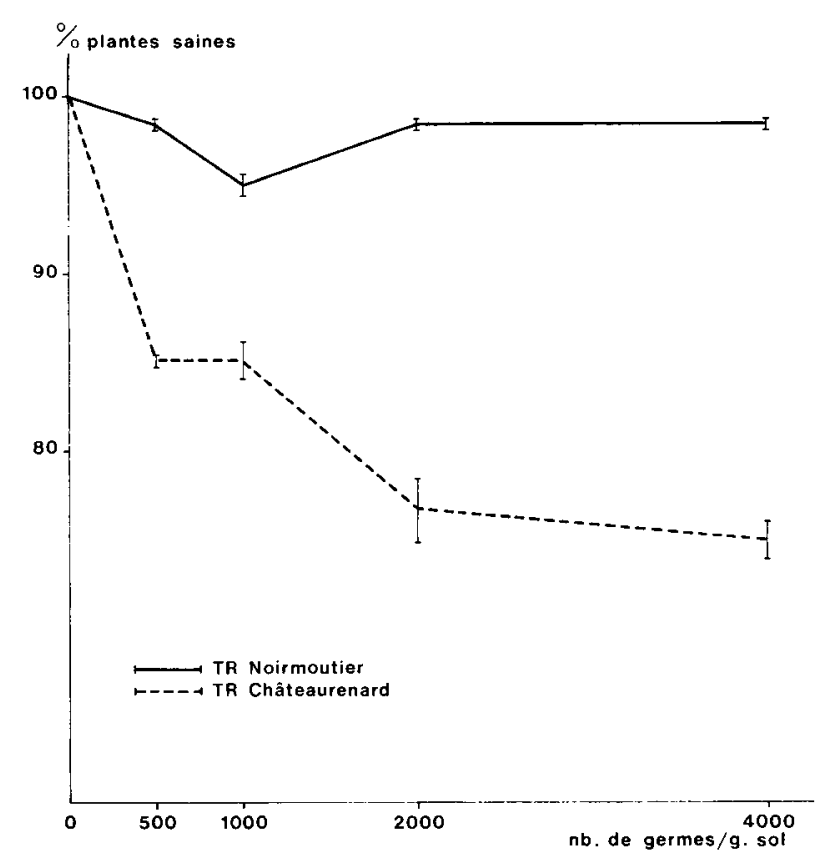

Figure 1

Comparaison des niveaux de résistance à la fusariose vasculaire du lin des sols de Noirmoutier et Châteaurenard: pourcentages de plantes saines 3 mois après infestation des sols avec des doses croissantes de $\mathrm{F}$. o. f. sp. lini (T.R. = terre résistante) .

Comparison of the levels of suppressiveness to fusarium-wilt of flax of the soils from Noirmoutier and Châteaurenard: percentages of healthy plants 3 months after soil infestation with increasing concentrations of $\mathrm{F}$. o. f. sp. lini (T.R. = suppressive soil).

\section{B. Nature microbiologique de la résistance du sol}

La figure 2 montre que le traitement thermique du sol $\left(100{ }^{\circ} \mathrm{C}, 30 \mathrm{mn}\right)$ détruit sa résistance et qu'il suffit d'introduire 10 p. 100 de sol résistant non traité pour rétablir la résistance dans le sol traité. Ces 2 observations suffisent à démontrer que la résistance de ce sol repose fondamentalement sur des interactions microbiennes. Elle est en effet détruite à la suite de l'élimination des microorganismes par le traitement à la chaleur (tabl. 2) et restaurée par introduction des microorganismes dans le sol désinfecté.

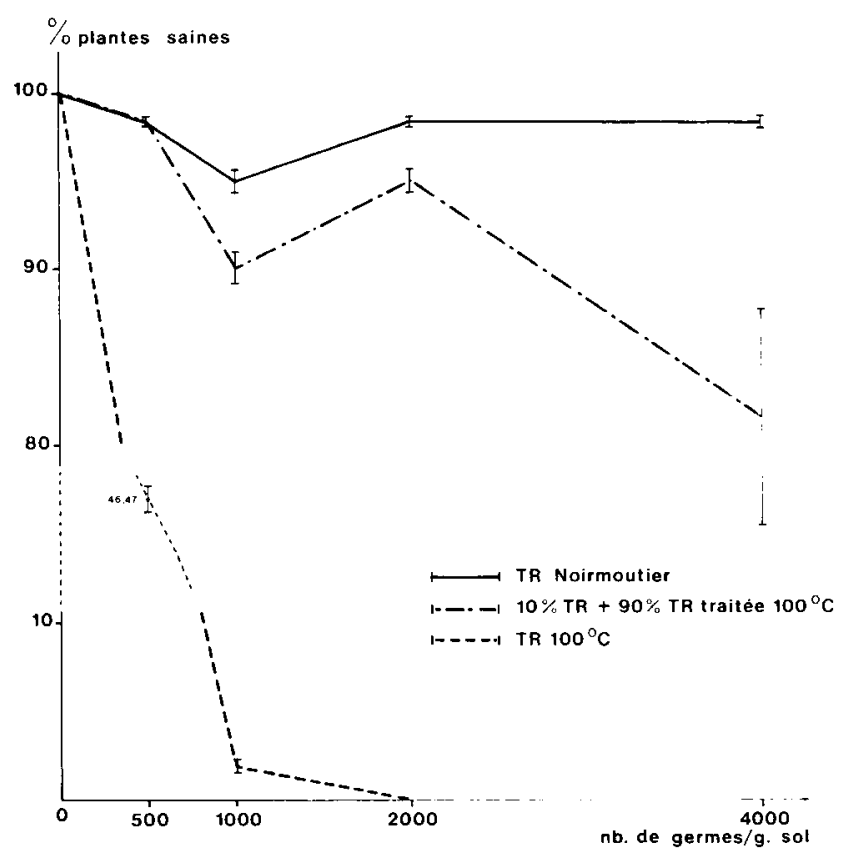

Figure 2

Réceptivité à la fusariose vasculaire du lin du sol de Noirmoutier traité à la chaleur $\left(100{ }^{\circ} \mathrm{C}, 30 \mathrm{mn}\right)$ et d'un mélange $10 \mathrm{p} .100 \mathrm{de}$ sol résistant $+90 \mathrm{p} .100$ de sol résistant traité à la chaleur : pourcentages de plantes saines 3 mois après infestation des sols avec des doses croissantes de F. of. sp. lini.

Receptivity to fusarium-wilt of flax of the soil from Noirmoutier after a heat-treatment $\left(100^{\circ} \mathrm{C}, 30 \mathrm{mn}\right)$ and of a mixture of 10 parts of suppressive soil with 90 parts of steamed suppressive soil : percentages of healthy plants 3 months after soil infestation with increasing concentrations of $\mathrm{F}$. o. $f$. sp. lini.

\section{Microorganismes responsables de la résistance}

L'application de traitements thermiques ménagés à 60 et $80^{\circ} \mathrm{C}$ a permis de montrer que la résistance est déjà détruite à la suite du traitement à $60^{\circ} \mathrm{C}$. Le tableau 2 indique les densités des populations microbiennes mises en évidence par analyse microbiologique après application de ces traitements. Le traitement à $60^{\circ} \mathrm{C}$, qui abaisse de manière importante la densité de la population fongique, détruit totalement les populations de Pseudomonas fluorescents et de Fusarium spp. Il n'est donc pas permis de différencier le rôle

TABLEAU 2

Densités des populations microbiennes analysées dans le sol de Noirmoutier, avant et après traitement à la chaleur, exprimées en milliers de germes $/ g$ de sol sec.

Microbial population densities determined before and after heat treatment of the soil from Noirmoutier, expressed in thousand C.F.U. per $g$ of dry soil.

\begin{tabular}{lrrrrr}
\hline \hline & Actinomycètes & Bactéries & $\begin{array}{c}\text { Pseudomonas } \\
\text { fluorescents }\end{array}$ & Champignons & Fusarium \\
\hline non traité & 146 & 12930 & 380 & 116 & 6 \\
$60^{\circ} \mathrm{C}, 30 \mathrm{mn}$ & 46 & 11960 & 0 & 0 & 0 \\
$80^{\circ} \mathrm{C}, 30 \mathrm{mn}$ & 0 & 5180 & 0 & 0 \\
$100^{\circ} \mathrm{C}, 30 \mathrm{mn}$ & 0 & 2 & 0 & 0 \\
\hline \hline
\end{tabular}


joué par chacune de ces 2 populations. Pour le faire il convient d'étudier l'aptitude de ces microorganismes à induire la résistance dans le sol préalablement traité à la chaleur.

La figure 3 montre que l'introduction en mélange de 5 souches de $F$. oxysporum non pathogènes permet d'établir un très bon niveau de résistance dans le sol traité à la chaleur alors que l'introduction en mélange de 7 souches de Pseudomonas fluorescents n'a qu'un effet très limité. Dans les 2 cas, les populations introduites se sont bien installées dans le sol, leur densité s'établissant à $7.10^{8}$ et $8.10^{5}$ germes $/ g$ de sol respectivement pour les Pseudomonas et les Fusarium. L'addition simultanée des Pseudomonas et des Fusarium ne se traduit pas par un effet synergique, le niveau de résistance observé étant comparable à celui déterminé par les seuls Fusarium.

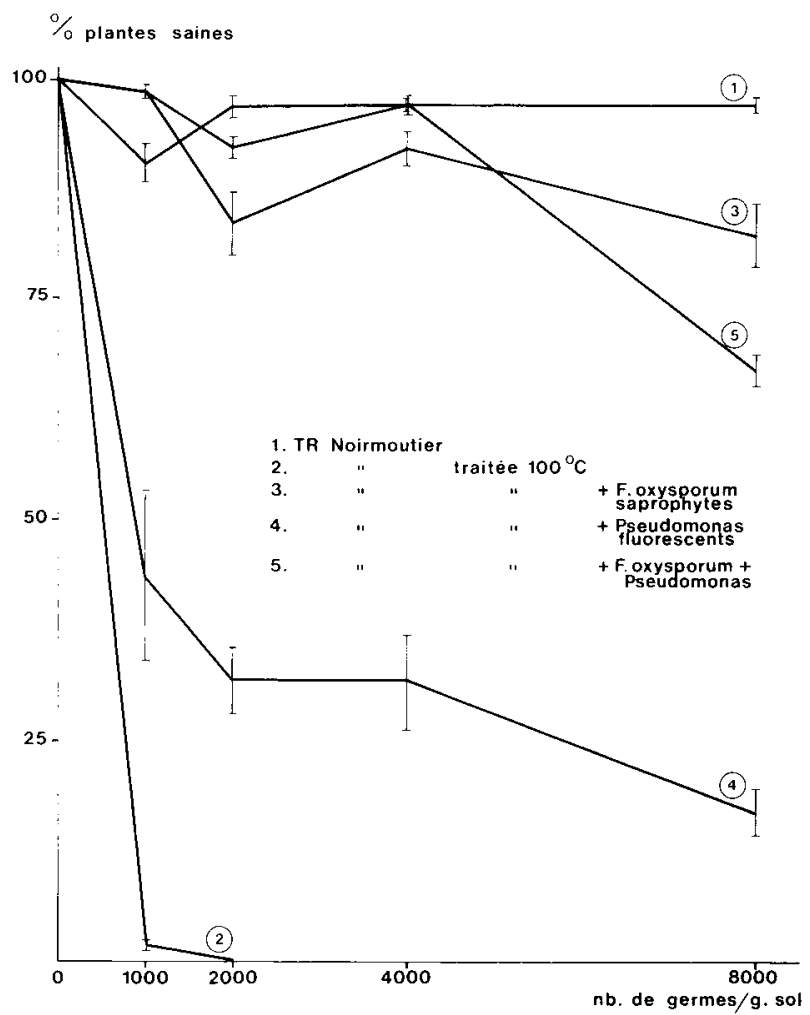

Figure 3

Niveau de réceptivité du sol de Noirmoutier traité à la chaleur puis recolonisé par différentes souches de Fusarium oxysporum non pathogènes et de Pseudomonas fluorescents : pourcentage de plantes saines 3 mois après infestation des sols avec des doses croissantes de F. o. $f$. sp. lini.

Level of receptivity of the soil from Noirmoutier, heat-treated and recolonized by different strains of non-pathogenic Fusarium oxysporum or fluorescent Pseudomonas : percentage of healthy plants 3 months after soil infestation with increasing concentrations of F. o. $f$. sp. lini.

La figure 4 montre que l'apport de Fe E.D.T.A. $(200 \mu \mathrm{g} / \mathrm{g})$ n'a aucun effet sur le niveau de résistance du sol, indiquant que la compétition pour le fer, exercée par les Pseudomonas, n'est pas un facteur important dans ce sol résistant. L'apport de glucose contribue au contraire à abaisser le niveau de résistance de ce sol, de manière très modérée (10 p. 100 environ) mais significative.

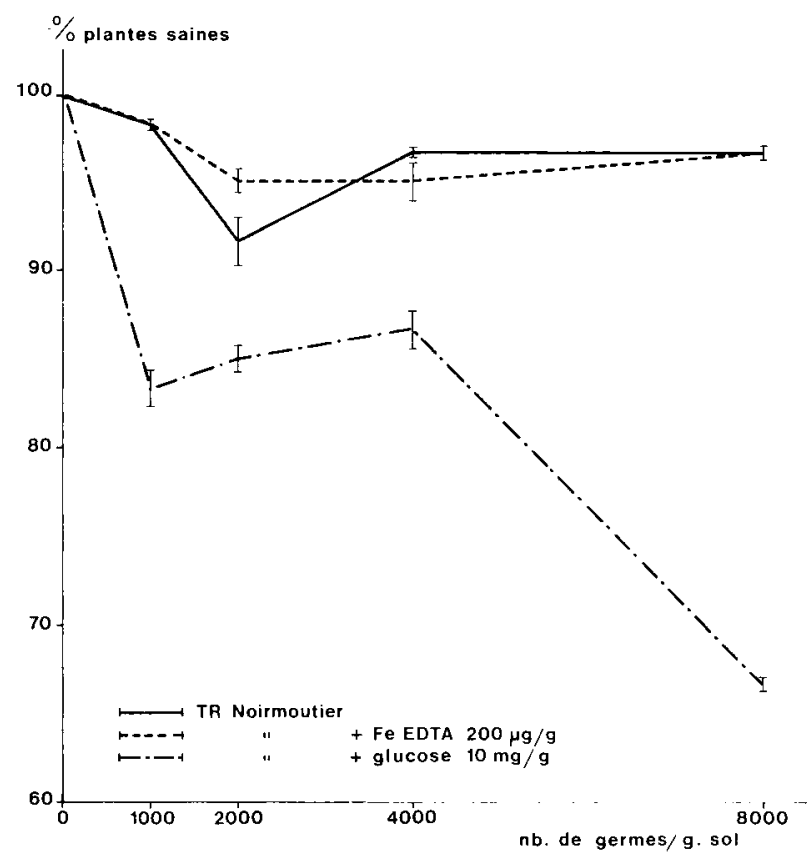

Figure 4

Niveau de réceptivité du sol de Noirmoutier amendé avec du glucose $(10 \mathrm{mg} / \mathrm{g})$ ou du Fe E.D.T.A. $(200 \mu \mathrm{g} / \mathrm{g})$ : pourcentage de plantes saines après infestation des sols avec des concentrations croissantes de F. o. f. sp. lini.

Level of receptivity of the soil from Noirmoutier after addition of glucose $(10 \mathrm{mg} / \mathrm{g}$ soil) or Fe E.D.T.A. $(200 \mu \mathrm{g} / \mathrm{g})$ : percentage of healthy plants 3 months after soil infestation with increasing concentrations of F. o. $f$. sp. lini.

Par analogie avec les résultats enregistrés pour le sol de Châteaurenard (AlABOUvetTE et al., 1985a), ces observations indiquent que les Fusarium non pathogènes jouent un rôle important dans ces mécanismes de résistance.

\section{Aptitudes de différentes souches de Fusarium à induire la résistance des sols}

L'analyse microbiologique, réalisée sur le milieu sélectif de KOMADA, révèle que la population de $F u s a-$ rium spp. est dans ce sol particulièrement élevée :

$$
\frac{F . \text { oxysporum }}{15700 \pm 2500} \quad \frac{\text { F. roseum }}{15500 \pm 2300} \quad \frac{\text { F. solani }}{4600 \pm 660}
$$

L'expérimentation précédente ayant démontré que les $F$. oxysporum sont capables de rétablir la résistance dans le sol préalablement traité à la chaleur, il convient d'évaluer le rôle joué par les autres espèces de Fusarium dans les mécanismes de résistance de ce sol.

Trente souches de chacune des espèces $F$. oxysporum, $F$. roseum et $F$. solani ont été isolées du sol résistant puis réintroduites dans le sol préalablement traité à la chaleur et confrontées au $F$. oxysporum pathogène afin de déterminer leur aptitude à rendre le sol résistant. La figure 5 indique que les souches de $F$. oxysporum s'avèrent globalement beaucoup plus efficaces que les souches de $F$. roseum ou de $F$. solani. 


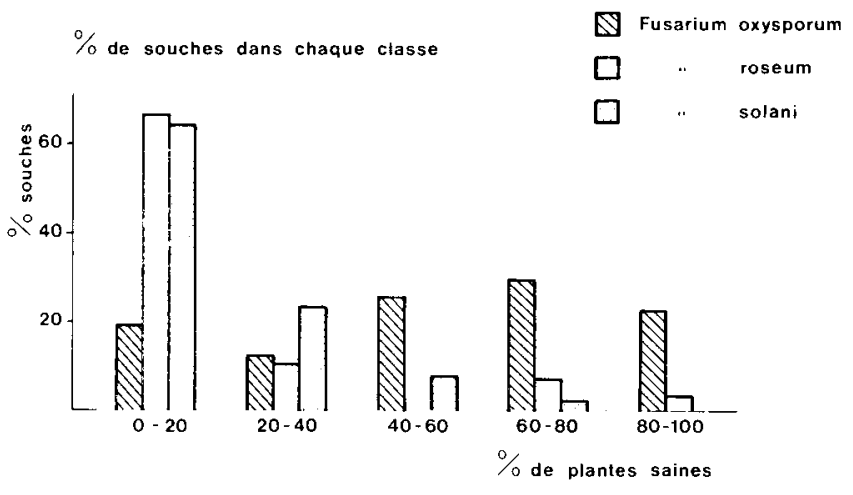

Figure 5

Aptitude de différentes souches de Fusarium oxysporum, F. roseum et $\mathrm{F}$. solani à rétablir la résistance dans le sol de Noirmoutier préalablement traité à la chaleur : répartition des souches dans 5 classes correspondant au pourcentage de plantes saines 6 semaines après infestation du sol avec F. o. f. sp. lini à la dose 2000 germes $/ g$ de sol.

Ability of different strains of $\mathrm{F}$. oxysporum, F. roseum and F. solani to reestablish the suppressiveness in the steamed suppressive soil: distribution of the strains in 5 classes corresponding to percentage of healthy plants 6 weeks after soil infestation with F. o. f. sp. lini at the concentration of 2000 C.F.U./g soil.

Cependant il existe une grande variabilité parmi les souches de $F$. oxysporum; il est donc important d'en étudier le comportement afin de déterminer leur mode d'action.

Les analyses microbiologiques réalisées montrent que toutes les souches de $F$. oxysporum introduites ont parfaitement colonisé le sol préalablement traité à la chaleur; le niveau des populations après 3 semaines d'incubation étant compris entre 20 et $50.10^{4}$ germes $/ \mathrm{g}$ de sol.

La méthode d'analyse employée ne permet pas de comparer statistiquement ces valęurs mais il est clair

TABLEAU 3

Comparaison entre l'aptitude de différentes souches de $\mathrm{F}$. oxysporum à coloniser le sol de Noirmoutier préalablement traité à la chaleur et leur aptitude à protéger une culture :

- densité de la population de Fusarium non pathogènes au moment de l'apport de l'agent pathogène et du semis ;

- pourcentage de plantes saines après 6 semaines de culture.

Comparison of the ability of different strains of F. oxysporum to colonize the heat-treated soil from Noirmoutier with their ability to control the disease:

- density of the population of non-pathogenic Fusarium at planting, when the pathogenic Fusarium is added;

- percentage of healthy plants 6 weeks after planting.

\begin{tabular}{ccc}
\hline $\begin{array}{c}\text { Souche } \\
\mathrm{N}^{\circ}\end{array}$ & $\begin{array}{c}\text { Densité de la } \\
\text { population }\end{array}$ & $\begin{array}{c}\text { Pourcentage de } \\
\text { plantes saines }\end{array}$ \\
\hline 5 & $470.10^{3}$ & 92 \\
6 & $470.10^{3}$ & 90 \\
7 & $360.10^{3}$ & 84 \\
8 & $430.10^{3}$ & 82 \\
1 & $310.10^{3}$ & 72 \\
20 & $520.10^{3}$ & 68 \\
34 & $670.10^{3}$ & 65 \\
11 & $420.10^{3}$ & 58 \\
47 & $550.10^{3}$ & 33 \\
4 & $470.10^{3}$ & 19 \\
3 & $300.10^{3}$ & 4 \\
\hline
\end{tabular}

que certaines souches présentant des niveaux de population comparables n'ont pas induit des niveaux de résistance identiques (tabl. 3 ).

D'autre part, l'étude de la colonisation des racines de lin cultivées dans le sol colonisé par diverses souches de $F$. oxysporum non pathogènes n'a pas permis de mettre en évidence de différences significatives dans l'aptitude des souches à coloniser les racines. Il n'a donc pas été possible d'établir une corrélation entre le taux de colonisation des racines et l'aptitude des souches à rendre le sol résistant (tabl. 3 et 4 ).

Enfin, la préinoculation de plantules de tomate avec une suspension de conidies de $F$. oxysporum indique que certaines souches possèdent dans ces conditions l'aptitude à diminuer la gravité de la maladie. Le tableau 5 montre que cette aptitude, souvent mise en évidence pour les formes pathogènes non compatibles avec la plante-hôte utilisée et attribuée aux phénomè-

\section{TABLEAU 4}

Taux de colonisation des racines de plantules de lin âgées de $15 j$ et cultivées dans le sol de Noirmoutier traité à la chaleur puis recolonisé par différentes souches de F. oxysporum non pathogènes, préalablement isolées du même sol.

Root colonization of 15-day old flax seedlings grown in the heattreated soil from Noirmoutier colonized by different strains of nonpathogenic $\mathrm{F}$. oxysporum, previously isolated from the same soil.

\begin{tabular}{rc}
\hline $\begin{array}{c}\text { Souche } \\
N^{\circ}\end{array}$ & $\begin{array}{c}\text { Nombre de colonies } \\
\text { de Fusarium } / \mathrm{cm} \text { de racine }\end{array}$ \\
5 & $0,54 \pm 0,39$ \\
10 & $0,37 \pm 0,30$ \\
8 & $0,59 \pm 0,38$ \\
11 & $0,34 \pm 0,13$ \\
9 & $0,33 \pm 0,20$ \\
\hline
\end{tabular}

\section{TABLEAU 5}

Effet protecteur obtenu par la préinoculation de plantules de tomate par différentes souches de $\mathrm{F}$. oxysporum non pathogènes : pourcentages de plantes saines 11 semaines après l'inoculation de F. o. $f . s p$ lycopersici. Les moyennes suivies d'une même lettre ne sont pas significativement différentes au seuil de 5 p. 100 d'après le test de Duncan.

Cross protection due to pre-inoculation of tomato seedlings with different strains of non-pathogenic $\mathrm{F}$. oxysporum : percentages of healthy plants 11 weeks after inoculation with $\mathrm{F}$. o. $f$. sp. lycopersici.

\begin{tabular}{cc}
$\begin{array}{c}\text { Souches } \\
\mathrm{N}^{\circ}\end{array}$ & $\begin{array}{c}\text { Pourcentage } \\
\text { de plantes saines }\end{array}$ \\
\hline
\end{tabular}

$F$. oxysporum f. sp. dianthi
$F$. oxysporum f. sp. melonis
$F$. oxysporum f. sp. vasinfectum
$F$. oxysporum f. sp. lini
$F$. oxysporum f. sp. lycopersici
$F$. oxysporum non pathogène 5
$F$. oxysporum non pathogène 9
$F$. oxysporum non pathogène 1
$F$. oxysporum non pathogène $10 \mathrm{a}$
$F$. oxysporum non pathogène 11
$F$. oxysporum non pathogène $8 \mathrm{a}$
$F$. oxysporum non pathogène 47
$F$. oxysporum non pathogène $7 \mathrm{a}$
$F$. oxysporum non pathogène 10
$F$. oxysporum non pathogène $\mathrm{N} 1$
$F$. oxysporum non pathogène $\mathrm{N} 2$
63,3 a 43,8 a b 25,0 b c d e 20,0 b c d e 0 43,3 a b c 27,5 a b c d 18,3 b c d e 28,8 b c d e 28,8 b c d e 13,3 c d e 11,3 d e 8,3 d e 5,0 e 0 e

0 
nes de prémunition, se manifeste également pour quelques souches non pathogènes étudiées au cours de cette expérimentation.

Bien que très partielle, cette expérimentation suggère que les $F$. oxysporum possèdent différents modes d'action susceptibles de concourir à la protection des cultures contre les fusarioses vasculaires.

\section{DISCUSSION}

La mise au point d'une technique standardisée d'évaluation du niveau de réceptivité des sols aux fusarioses vasculaires (ALABOUVETTE et al., 1982) permet de vérifier au laboratoire dans des conditions parfaitement reproductibles la validité des observations réalisées dans les cultures et relatives à la résistance de certains sols aux fusarioses vasculaires. Ainsi nous avons pu établir que ce sol sableux de Noirmoutier possède un niveau très élevé de résistance aux fusarioses vasculaires.

L'application à l'étude de ce sol de la méthodologie mise au point lors des recherches consacrées à la résistance des sols de Châteaurenard (LOUVET et al., 1981) a permis de progresser rapidement dans la connaissance des propriétés de la résistance du sol de Noirmoutier. Les résultats acquis démontrent que cette résistance est fondamentalement de nature microbiologique comme celle des sols de Châteaurenard (LOUVET et al., 1976) de Salinas Valley (SCHER \& BAKER, 1980) et d'Albenga (TAMIETTI \& MATTA, 1984). Cependant on ne peut exclure le rôle de certains facteurs abiotiques, le pH de ces sols étant toujours supérieur à 7 .

SCHER \& BAKER (1982) ayant démontré que, dans les sols à $\mathrm{pH}$ élevé, la compétition pour le fer est un des facteurs qui limite l'activité des Fusarium oxysporum pathogènes, il convenait de vérifier l'existence de ce phénomène dans le sol de Noirmoutier. L'apport de Fe E.D.T.A. à une concentration supérieure à celle employée par SCHER \& BAKER (1982) n'a pas permis de briser la résistance du sol de Noirmoutier contrairement aux résultats acquis par ces auteurs avec le sol de Salinas. Cette observation est confortée par le fait que l'apport massif de Pseudomonas fluorescents, bactéries capables d'immobiliser le fer grâce à la production de sidérophores, n'a pas permis de rendre résistant le sol préalablement traité à la chaleur. On peut donc conclure que les Pseudomonas fluorescents ne jouent pas un rôle important dans les mécanismes de résistance de ce sol aux fusarioses vasculaires.

Comme le sol de Châteaurenard, le sol de Noirmoutier héberge une importante population fusarienne et l'introduction massive de $F$. oxysporum non pathogènes suffit à rétablir la résistance dans le sol préalablement traité à la chaleur. Ces 2 observations permettent de penser que les $F$. oxysporum autochtones jouent un rôle primordial dans les mécanismes de résistance de ce sol de Noirmoutier. Mais il n'est pas exclu que d'autres microorganismes également détruits par les traitements thermiques puissent jouer un rôle dans les mécanismes de résistance.
Trois hypothèses sont proposées pour expliquer le mode d'action des $F$. oxysporum non pathogènes. ALABOUVETTE et al. $(1983,1985)$ suggèrent que Fusarium pathogènes et non pathogènes entrent en compétition dans le sol pour certains éléments nutritifs indispensables et, en particulier, pour le carbone. SCHNEIDER (1984) pense que cette compétition intragénérique se déroule à la surface de la racine pour la conquête des sites d'infection. Cet auteur oppose ainsi la compétition parasitaire pour la colonisation de la racine à la compétition saprophytique qui se déroule dans le sol. Enfin, il est bien connu (MAS, 1967 ; MATTA, 1966 ; OGAWA \& KOMADA, 1984) que des $F$. oxysporum pathogènes placés au contact des racines d'une plante non compatible induisent des relations de résistance retardant l'apparition des symptômes. Les expérimentations rapportées dans cet article ne permettent pas de préciser l'importance relative de ces différents mécanismes dans le phénomène de résistance des sols.

Ainsi, l'apport de glucose, qui a pour but d'atténuer l'intensité de la compétition nutritive pour le carbone, n'a pas eu, dans le sol de Noirmoutier, un effet aussi net que celui observé dans le sol de Châteaurenard. Cependant, ayant démontré que l'effet d'un apport énergétique dépend à la fois de la biomasse microbienne et du niveau de la population fusarienne considérée (AlABOUVETTE et al., 1985b), il convient de poursuivre cette expérimentation en comparant l'efficacité de différentes concentrations de glucose apportées au sol de Noirmoutier. Mais il est vraisemblable que les Fusarium entrent également en compétition pour d'autres éléments nutritifs. Les résultats acquis jusqu'à présent ne permettent donc ni de confirmer ni de réfuter l'hypothèse de relations de compétition nutritive entre formes pathogènes et formes non pathogènes de $F$. oxysporum.

Il est de même difficile de porter un jugement définitif à partir des résultats concernant la colonisation des racines de lin par quelques souches de $F$. oxysporum non pathogènes. Il est nécessaire d'améliorer les techniques permettant d'estimer la colonisation racinaire par les $F$. oxysporum et de comparer des souches ayant des caractéristiques très différentes quant à leur aptitude à induire la résistance des sols. De plus, il serait utile de mieux connaître les processus d'infection des plantes par les $F$. oxysporum agents de trachéomycose afin de savoir reconnaître les sites d'infection.

Enfin, les résultats présentés indiquent que certaines souches de $F$. oxysporum non pathogènes sont, au même titre que certaines formes spéciales de $F$. $o x y$ sporum pathogènes, capables d'induire chez la tomate des mécanismes de résistance qui retardent l'apparition des symptômes. Remarquons qu'il est à l'heure actuelle impossible de différencier une souche purement saprophyte d'une souche pathogène sur un hôte différent de celui auquel elle est confrontée. Il est donc possible que certaines souches non pathogènes isolées du sol résistant soient en réalité des formes spéciales impossibles à déterminer en absence de leur hôte spécifique. Mais, indépendamment de cette question, il est peu vraisemblable que ces phénomènes de résistance induite qui ne font que retarder l'infection soient à eux seuls responsables de la résistance des sols qui se manifeste tout au long de la culture. Il est 
cependant possible qu'ils participent aux mécanismes de résistance des sols, par exemple en abaissant la sensibilité de la plante-hôte.

La compréhension des phénomènes de résistance des sols aux fusarioses vasculaires nécessite donc la poursuite et l'élargissement des recherches aussi bien dans le domaine des relations plante-agent pathogène et plante-microorganisme non pathogène que dans celui de l'écologie microbienne des sols.

Reçu le Ier octobre 1985 Accepté le 18 février 1986

\section{RÉFÉRENCES BIBLIOGRAPHIQUES}

Alabouvette C., Couteaudier Y., Louvet J., 1982. Comparaison de la réceptivité de différents sols et substrats de culture aux fusarioses vasculaires. Agronomie, 2 (1), 1-6.

Alabouvette C., Couteaudier Y., Louvet J., 1983. Importance des phénomènes de compétition nutritive dans l'antagonisme entre microorganismes, 7-16. In "Les antagonismes microbiens. Modes d'action et application à la lutte biologique contre les maladies des plantes ». 24e Coll. soc. Fr. Phytopathol., Bordeaux, 26-28 mai $1983,360 \mathrm{p}$.

Alabouvette C., Couteaudier Y., Louvet J., 1985a. Recherches sur la résistance des sols aux maladies. XI. - Etude comparative du comportement des Fusarium spp. dans un sol résistant et un sol sensible aux fusarioses vasculaires enrichis en glucose. Agronomie, 5 (1), 63-68.

Alabouvette C., Couteaudier Y., Louvet J., 1985b. Recherches sur la résistance des sols aux maladies. XII. - Activité respiratoire dans un sol résistant et un sol sensible aux fusarioses vasculaires enrichis en glucose. Agronomie, 5 (1), 69-72.

Arjunarao V., 1971. Biological control of cotton wilt. II. - In vivo effect of antagonists on the pathogen Fusarium vasinfectum. Proc. Indian Acad. Sci., Sect. B, 74, 53-62.

Cook R. J., Baker K. F., 1983. The nature and practice of biological control of plant pathogens. Am. Phytopathol. Soc., St Paul MN, USA, $539 \mathrm{p}$.

Komada H., 1975. Development of a selective medium for quantitative isolation of Fusarium oxysporum from natural soil. Rev. Plant Prot. Res., 8, 114-125.

Louvet J., Rouxel F., Alabouvette C., 1976. Recherches sur la résistance des sols aux maladies. I. - Mise en évidence de la nature microbiologique de la résistance d'un sol au développement de la fusariose vasculaire du melon. Ann. Phytopathol., 8, 425-426.

Louvet J., Alabouvette C., Rouxel F., 1981. Microbiological suppressiveness of some soils to Fusarium wilts, 262-275. In P. E. Nelson, T. A. Tousson, R. J. Cook : « Fusarium : Diseases, Biology and Taxonomy ». The Pa. State Univ. Press, $457 \mathrm{p}$.

Mas P., 1967. Protection du melon contre la fusariose par infection préalable de la plantule avec d'autres souches de Fusarium. C. $R$. Acad. Agric. Fr., 53, 1034-1040.
Matta A., 1966. Effeto immunizante di alcuni micromiceti verso le infezioni su $F$. oxysporum f. lycopersici su pomodoro. Ann. Fac. Sci. Agr. Univ. Torino, 3, 85-98.

Ogawa K., Komada H., 1984. Biological control of Fusarium wilt of sweet potato by non-pathogenic $F$. oxysportum. Ann. phytopath. Soc. Jap., 50, 1-9.

Rouxel F., Bouhot D., 1971. Recherches sur l'écologie des champignons parasites dans le sol. IV. Nouvelles mises au point concernant l'analyse sélective et quantitative des Fusarium oxysporum et Fusarium solani dans le sol. Ann. Phytopathol., 3 (2), 171-188.

Rouxel F., Alabouvette C., Louvet J., 1979. Recherches sur la résistance des sols aux maladies. IV : Mise en évidence du rôle des Fusarium autochtones dans la résistance d'un sol à la fusariose vasculaire du melon. Ann. Phytopathol., 11, 199-207.

Scher M. F., Baker R., 1980. Mechanisms of biological control in a Fusarium suppressive soil. Phytopathology, 70, 412-417.

Scher M. F., Baker R., 1982. Effects of Pseudomonas putida and synthetic iron chelator on induction of soil suppressiveness to Fusarium wilt pathogens. Phytopathology, 72, 1567-1573.

Schneider R. W., 1982. Suppressive soils and plant disease. $A m$. Phytopathol. Soc., St Paul Mn, USA, 87 p.

Schneider R. W., 1984. Effects of non-pathogenic strains of Fusarium oxysporum on celery root. Infection by Fusarium oxysporum f. sp. apii and a novel use of the Lineweaver Burk double reciprocal plot technique. Phytopathology, 14, 646-653.

Stotzky G., 1967. Clay minerals and microbial ecology. Trans. N.Y. Acad. Sci., 30, 11-21.

Stover R. H., 1956. Studies on Fusarium wilt of bananas. I. The behavior of $F$. oxysporum f. cubense in different soils. Can. $J$. Bot., 34, 927-942.

Tamietti G., Matta A., 1984. Fusarium lycopersici suppressive properties in the soil of Albenga (Italy) ; preliminary observations. Proc. 6th Congr. Union Phytopathol. Mediterr. Cairo, Egypt, 129 131.

Tu C. C., Cheng Y. H., 1981. Soil microbial activity in relation to Fusarium wilt suppressive soil and conducive soil. Taiwan Dais Sci. Meet. Rep., 1-12. 